\title{
BMJ Open Quality Assessment and improvement of junior doctor handover in the emergency department
}

\author{
Mark Sykes, ${ }^{1}$ Jack Garnham, ${ }^{2}$ Pablo Martin Kostelec, ${ }^{3}$ Hazel Hall, ${ }^{2}$ Anu Mitra ${ }^{2}$
}

To cite: Sykes M, Garnham J, Kostelec PM, et al. Assessment and improvement of junior doctor handover in the emergency department. BMJ Open Quality 2020;9:e001032. doi:10.1136/ bmjoq-2020-001032

- Additional material is published online only. To view, please visit the journal online (http://dx.doi.org/10.1136/ bmjoq-2020-001032).

Received 2 June 2020 Revised 21 July 2020 Accepted 27 July 2020

\section{Check for updates}

(C) Author(s) (or their employer(s)) 2020. Re-use permitted under CC BY-NC. No commercial re-use. See rights and permissions. Published by BMJ.

${ }^{1}$ Trauma and Orthopaedic Surgery, Guy's and St Thomas' NHS Foundation Trust, London, UK

${ }^{2}$ Emergency Department,

Charing Cross Hospital, London, UK

${ }^{3} \mathrm{NHS}$ Improvement, London, UK

Correspondence to

Mark Sykes;

marksykes@nhs.net

\begin{abstract}
Introduction Effective handover between junior doctors is widely accepted as essential for patient safety. The British Medical Association in association with the National Health Service (NHS) National Patient Safety Agency and NHS Modernisation Agency have produced clear guidance regarding the contents and setting for a safe and efficient handover. We aimed to understand current junior doctor's opinions on the handover process in a London emergency department (ED), with subsequent assessment, and any necessary improvement, of handover practices within the department.
\end{abstract}

Methods In a London ED, a baseline survey was completed by the senior house officer (SHO) cohort to gauge current opinions of the existing handover process. Concurrently, a blinded prospective audit of handover practises was conducted. Multiple improvement strategies were subsequently implemented and assessed via Plan-Do-Study-Act (PDSA) cycles. A standard operating procedure was initially introduced and 'rolled out' throughout the department. This intervention was followed by development of an electronic handover note to ease completion of a satisfactory handover. Additional surveys were conducted to continually assess SHO opinion on how the handover process was developing. The final improvement strategy was formal handover teaching at the SHO induction.

Results Baseline audit and SHO survey highlighted several opportunities for improvement. 5 handover components were deemed essential: (1) documented handover note; (2) doctor's names; (3) history of presenting complaint; (4) ED actions; and (5) ongoing plan. The frequency of these components saw significant improvement by completion of the final PDSA. Following SHO rotation, all of the essential components fell, only to recover after the next improvement strategy.

Conclusions Junior doctors in a London ED were not satisfied with the current SHO handover process, and handover practices were not adequate. While the rotational nature of the $\mathrm{SHO}$ cohort makes sustained change challenging, implementation of thoughtful and realistic improvement strategies can significantly improve handover quality.

\section{PROBLEM}

Increasing pressure on emergency departments (EDs) has been well documented in recent years ${ }^{1}$ and is posing new challenges to healthcare professionals throughout the patient journey. With the shift-based nature of the ED senior house officer ( $\mathrm{SHO}$ ) rota, one such challenge is performing a documented, safe and efficient handover of patient care between outgoing and incoming SHOs. Traditionally in the form of a brief conversation and handwritten notes at the end of a shift, there is a risk that a handover can be incomplete and/or inaccurate with potential implications for patient safety. This is of particular concern when considering the impact of shift work and cognitive drop-off at the end of a shift. ${ }^{2}$ The advent of electronic medical records (EMRs) provides an opportunity to standardise handovers, ${ }^{3}$ introduce quality control and move away from what was historically an informal and risky processes.

This quality improvement project (QIP) was introduced in the ED of a busy London teaching hospital. Containing a hyperacute stroke unit, providing tertiary multispecialty care and facing the national increase in hospital attendances, the ED patient volume is high. At the time of writing, the department has capacity for 12 patient cubicles, 5 resuscitation bays, a large minor illness waiting area and reserve capacity for additional beds if required. As is commonplace among EDs, the SHO rota is staffed with a combination of foundation year 2 trainees, acute care common stem (ACCS) trainees, GP ST1 (General Practitioner Specialist Trainee 1) trainees, middle grade doctors and locum doctors, all of whom have varied experience from multiple backgrounds. Without specific handover guidance or local standardisation, understandably individual doctors had differing expectations regarding the contents and setting of a patient handover.

At our department, a formal multidisciplinary morning handover occurs daily at 08:00, with every member of night shift and day shift staff. No such formality exists, however, for handover of individual patients between SHO shifts. Considering the shift patterns, a normal day can offer multiple 


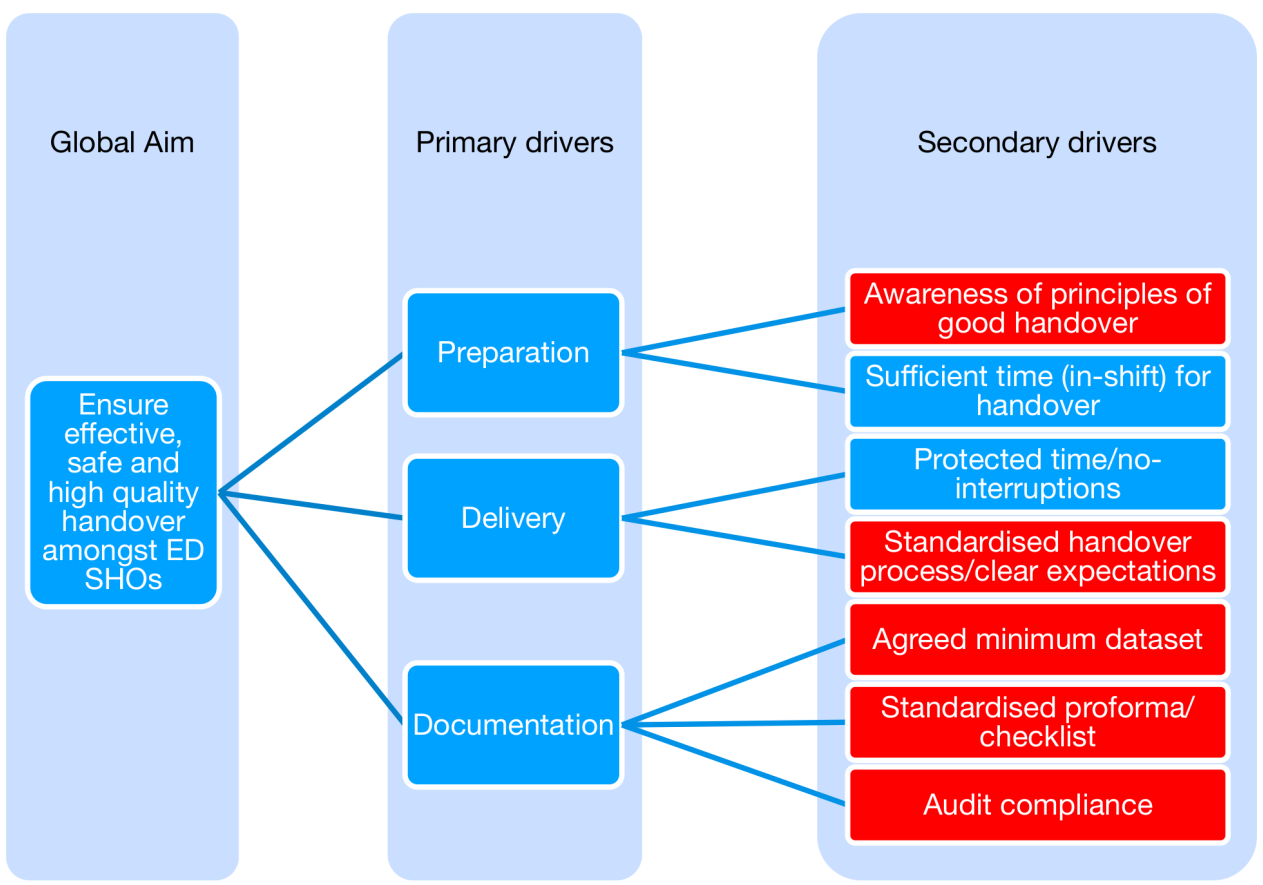

Figure 1 Effective and safe handover driver diagram. ED, emergency department; SHOs, senior house officers.

opportunities for patient handovers. High workload, lack of standardisation and differing doctor expectation led to handovers of variable quality resulting in additional and repeated work, impact on doctor morale and concerns regarding impact on patient safety

The global aim of this QIP was to ensure that the handover between outgoing and incoming SHOs in the ED was consistently safe and effective. A driver diagram, outlining primary and secondary drivers of effective handover, can be seen in figure 1 .

An anonymous survey among the cohort of ED SHOs, exploring their views of the end of shift handover process, had revealed significant improvement opportunities with the local handover process.

The average 'effectiveness' rating of the end of shift handover among the 13 SHOs questioned was 5/10, while the morning formal handover achieved a score of $8.1 / 10$. When asked about the 'accuracy' and 'comprehensiveness' of the end of shift informal handover, the average ratings were 5.5/10 and 4.5/10, respectively. Fifty-four per cent of doctors reported difficulties in patient care due to incomplete/inaccurate handovers, and when asked to rate how safe the current handover process is, the average score was 5.2/10. Free-text answers were requested to further understand why the ED SHOs responded in this way and to gain a consensus on options for improving the handover process. Additional questions regarding handover practice illustrated that handovers were predominantly to a doctor of the same grade. Importantly, no concerns regarding the practical ability to handover at the end of the shift, such as protected time during the rostered shift, were highlighted. Specifically, the frustrations were centred on the handover process itself.
In summary, a combination of high workload, lack of standardisation and differing doctor expectation had led to handovers of variable quality, resulting in duplication of work and negatively impacting on patient safety. All of this was having a negative impact on staff morale.

On review of the driver diagram, the decision was made to address the following aspects of handover:

- Awareness of principles of good handover.

- Standardisation of handover process.

- Handover documentation.

In order to be able to objectively evaluate handover, particular attention was paid to improving handover documentation as a proxy of actual handover practice.

\section{BACKGROUND}

Effective handover between junior doctors is widely accepted as a critical component of patient safety. The British Medical Association in association with the National Health Service (NHS) National Patient Safety Agency and NHS Modernisation Agency have produced clear guidance regarding the contents and setting for a safe and efficient handover. ${ }^{4}$ The Royal College of Physicians has also produced the 'Acute care toolkit' for handover, calling for standardisation of the handover process. ${ }^{5}$ There is evidence to suggest, however, that despite attempts to consolidate handover into an effective, timeefficient and safe process, variability in format and quality exists. $^{6-8}$

The drive to improve and standardise handovers has become well established in the literature as the focus of multiple QIP and literature reviews. These studies have largely addressed challenges in patient handover between inpatient medical shifts, often day-night handover, with 
few highlighting the issue in the ED. Similar to the concerns addressed through this QIP, interviews with ED consultants and middle-grade doctors in a similar London ED highlighted that the handover process needed improving. Prevailing concerns raised in this study were wasted doctor's time and negative impact on patient safety due to delays in treatment and investigation following inadequate handovers. ${ }^{9}$

Multiple strategies to improve the handover process have been documented in the literature. A common theme is standardisation of handover processes, which is echoed in the aforementioned national body publications. ${ }^{45}$ With increasing uptake of EMRs, this can be easily achieved with prepopulated electronic handover templates. Electronic handovers also make handover documentation easier to audit but perhaps most importantly reduce the risk of duplicated handwritten notes that are easily lost and are more likely to contain errors. ${ }^{10}$ In light of this, several studies have used either their preexisting EMR system ${ }^{811} 12$ or well-known packages such as Microsoft Excel ${ }^{6} 13-15$ to document the patient handover. Our institution employs the CERNER electronic health record software. ${ }^{16}$ While supporting ED care through medical alerts, centralisation of health records and several other features, there is not a built-in handover note on the CERNER system that we use in the ED. The software however is modifiable, and prepopulated handover notes can be added.

\section{BASELINE MEASUREMENT}

Guided by national published guidance, ${ }^{4}{ }^{17}$ a baseline unobserved prospective audit of 15 'handed over' patient's notes was completed assessing elements of the ED SHO patient handover. An anonymous survey was also completed by the ED SHOs to seek their perspectives on the current handover process.

The parameters to be audited were as follows:

- Documented handover note.

- Exchange of doctor's names on the electronic ED board.

- Documented history of presenting complaint (HPC).

- Documented ED actions up until handover.

- Documented handover plan.

The baseline handover audit was completed over a period of 3 weeks to ensure patient handovers were included from multiple different doctors in the ED. The baseline data collection confirmed concerns that the handover content was often inadequate, with only the "exchange of doctor's names on the electronic ED board being consistently performed. Results of the baseline audit can be seen in figure 2.

As illustrated in figure 2, no patient handovers during the 3-week data collection included a formal handover note, a documented history of presenting complaint or any information about progress/clinical interventions performed prior to handover. Exchange of doctor's names on the electronic ED board was expectedly high as

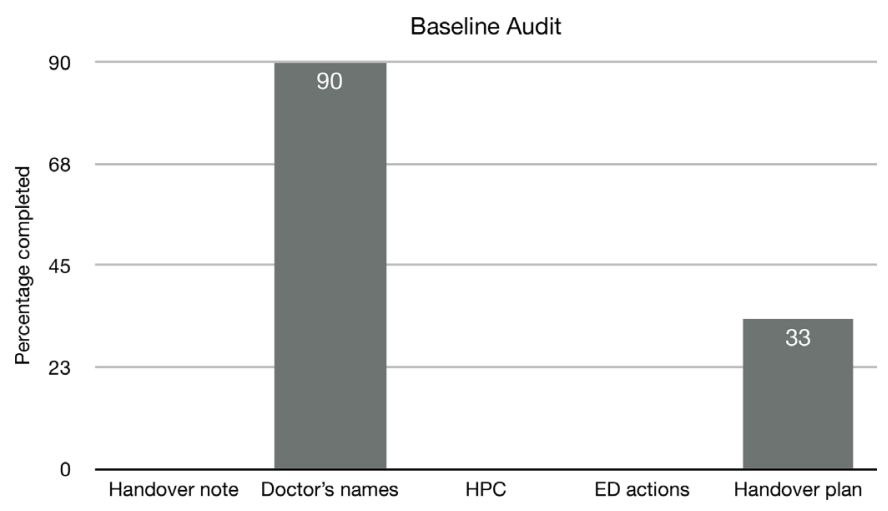

Figure 2 Baseline handover audit. Percentage completion for each audit standard is recorded. ED, emergency department.

this visual aid allows all ED staff to identify the doctor who is actively looking after specific patients; it is reinforced and often completed by the shift leader.

\section{DESIGN}

The baseline audit and associated survey demonstrated both objective and subjective cause for concern regarding existing SHO handover. The morning formal departmental handover was rated highly by the SHOs; however, the practicalities of achieving such a comprehensive meeting for every handed over patient is unrealistic, especially given the staggered end of shift times for ED SHOs. The qualitative questionnaire provided opinions on how the SHO handover could be improved; we also approached consultant and registrar colleagues to discuss potential improvement strategies. The common theme from these discussions was again one of standardisation. Additional discussion points were that of 'handing-up', meaning that all handed over patients are at minimum discussed with a more senior doctor, requests for formal teaching on handing over and a change in ethos to permit an uninterrupted handover period at the end of the SHO shift.

We therefore tested multiple improvement initiatives through Plan-Do-Study-Act (PDSA) cycles, allowing the impact of each intervention to be assessed individually. A summary of the strategies introduced is as follows:

1. Defined a departmental standard for the handover process between ED SHOs. Through staff discussions and use of published guidance, we were able to create a handover standard operating procedure (SOP), which included a 'handover checklist' that was signed off by the ED consultant body and introduced with electronic reminders and printed posters. The SOP defined the essential contents of handover and reminded ED SHOs to always handover to a more senior colleague through an uninterrupted handover meeting. Reminders to refer to the SOP were provided by the ED lead during each morning meeting.

2. Introduction of a prepopulated handover document on CERNER. CERNER permits the creation of prepop- 
ulated documents. We used this functionality to create a handover shortcut in line with the previously introduced SOP. This ensured that headings of required handover components were automatically present, providing a memory aid to the doctors performing handover. These templates could be activated and embedded into the notes by use of a shortcut phrase, for example, EDHO or H/over.

3. Introduction of a handover talk at departmental induction for each new cohort of SHOs. Responding to the request for formal teaching on handover, a session at induction allows the SOP and underpinning evidence to be explained. This also provides an opportunity to demonstrate the use of the CERNER handover note; to this end, the handover teaching was inserted into the CERNER training session.

\section{STRATEGY}

Through standardisation and more effective utilisation of the electronic health record system, we aimed to improve the effectiveness and safety of the SHO handover. The project was completed over an 18-month period, spanning through four rotations of ED SHOs. The rotational nature of the SHO rota makes consistent change more challenging and thus it was important to evaluate success of the project over a sustained period of time. We also hoped through our various improvement strategies that the SHO opinions on the handover process would become more positive.

\section{PDSA cycle 1}

Plan

Following the baseline measurement, the most striking realisation was the lack of formal documentation during the handover. The informality and apparent inaccuracy led to poor SHO opinions regarding the handover process. The aim of the first PDSA cycle was therefore to improve the documentation and quality of handovers through standardisation. We aimed to do this within a 4-month period. The success would be evaluated through repeat audit against national standards after implementation of the safety intervention.

\section{Do}

The first safety intervention to be introduced would be a handover checklist SOP (figure 3). A common theme from the baseline qualitative questionnaire was that a checklist would improve the handover process. With multiple doctors of different grades and from different backgrounds, the expectation of what a handover includes is likely to be very different and so introducing a standard checklist would alleviate this issue. The checklist was synthesised of essential but realistic handover components and was underpinned by published guidance. ${ }^{4} \mathrm{~A}$ prototype was discussed with and edited by consultant colleagues including the ED clinical lead, after which it was written into ED protocol as a handover SOP. The SOP was disseminated through multiple channels to

\section{ED Doctor Handover Checklist}

Following the recent staff questionnaire it is evident that important details are missed/ undocumented during the ED doctor handover. The following guide is an outline of what should be recorded on Cerner during the handover.

Essential documentation:

- Doctors' names

- Current patient situation

What has the patient presented with, brief history presenting complaint

- Actions taken within the ED

What has been done:

- Ireatmestigations completed and results

Handover plan

What is the receiving doctor asked to chase

Clear plan based on results

Which $\mathrm{SpR} /$ consultant is aware

\section{Handover rules:}

- For anything other than chasing results, hand up

- SpR or Consultant in charge should be aware of patient and handover

- Allocated doctor changed on Cerner

- Do not be interrupted during handover

- Make patient aware that care has been transferred

\section{Sate handover. sate patients. BMA, NHS modermisation agency, NHS national patient satety agency
SBAR. NHS institute tor innovation and improvement.}

Figure 3 Handover SOP. ED, emergency department; SOP, standard operating procedure. (SpR - Specialist Registrar)

ensure widespread coverage. An email to all ED medical staff, daily reminders at the formal morning meeting, reminders on the private ED SHO groups in social media and visual aids were used to introduce the SOP and reinforce its use.

\section{Study}

The handover SOP was used with the aforementioned reminders for approximately 1 month. Following this introductory period, a repeat prospective unobserved audit was conducted against the same published standards to assess the impact of the intervention. Results can be seen in figure 4 .

\section{Act}

The handover SOP had a significant impact on handover documentation. Achieving sustained improvement would be the next challenge as the 'roll out' of the SOP as was observed with the current cohort of SHOs would not be feasible for every subsequent rotation. A change in culture and practices needed to be ingrained in the ED.

\section{PDSA cycle 2}

Plan

In order to assess sustained improvement following the 4-monthly SHO change over, repeat prospective 


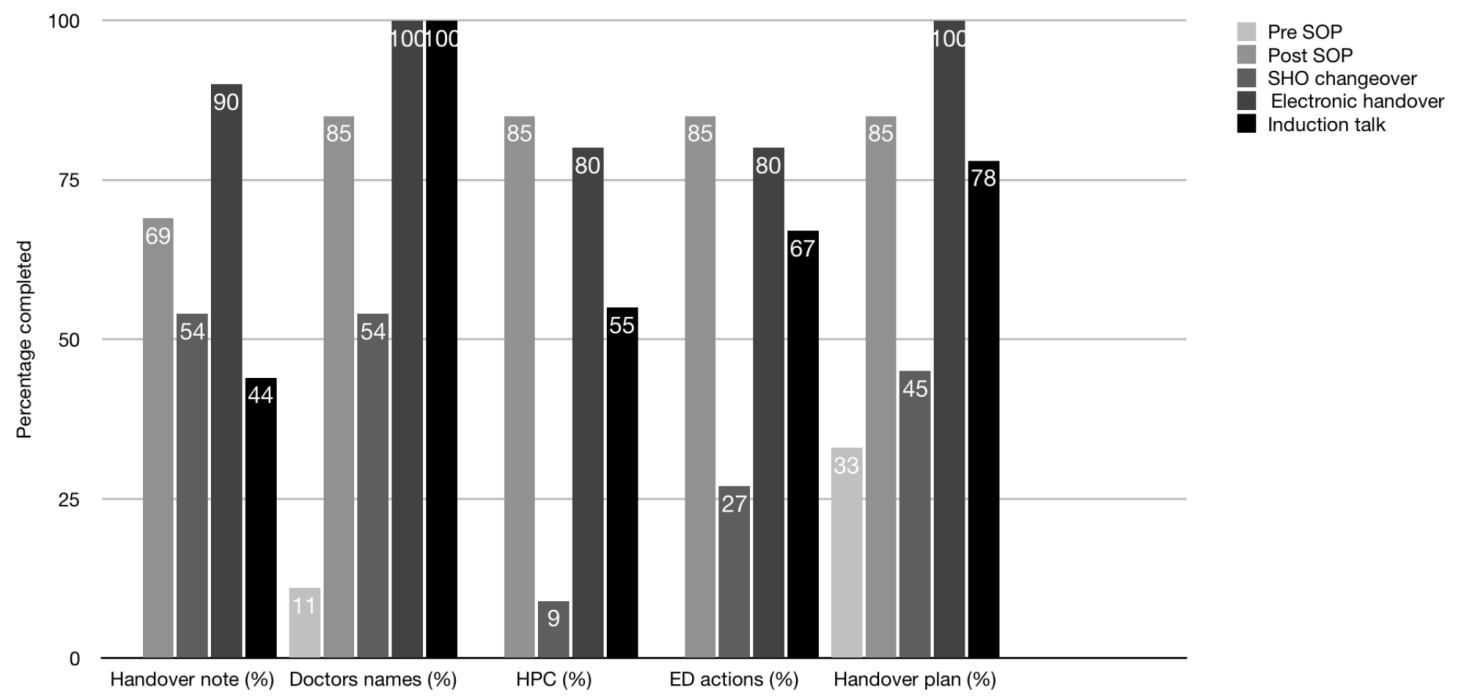

Figure 4 Impact of the improvement interventions on adherence to the handover SOP. SHO, senior house officer; SOP, standard operating procedure.

unobserved audit and subjective opinion poll was completed approximately 1 month into the new cohort's rotation. Following this we aimed to introduce an electronic prepopulated handover note on CERNER.

\section{Do}

As with the previous audit of handover documentation, an unobserved prospective audit was carried out. Alongside this, an opinion poll from the new ED SHO cohort was collected. An electronic handover note would then be introduced, populated in line with the handover SOP and thus providing an easy aide memoir to the contents of handover. Two options were considered to introduce an electronic handover note: (1) request the information technology (IT) department to create a handover note that can be selected alongside all other prepopulated documents on CERNER and (2) assist all ED SHOs in creating a handover shortcut themselves, which could be quickly accessed through typing a personalised shortcut word, for example, EDHO or H/over. On discussion with the IT department, it was likely to take several months to introduce a new document and therefore the immediate option of a handover shortcut was chosen. Each SHO was given a short training session by one of the QIP leads, during which the note was created. This ensured that the SHOs were familiar with how to use the handover shortcut but also confirmed consistency in the note adhering to the original handover SOP.

\section{Study}

As predicted, the audit following ED SHO changeover demonstrated a lapse in adherence to the handover SOP (figure 4). Additionally, there was concern from the new ED SHOs regarding the handover practice as illustrated in the repeat subjective opinion poll (online supplementary appendix 1). This disappointing but perhaps expected result can be interpreted as a lack of change in the ethos of handing over in the department. Following the introduction of the handover note shortcuts, a repeat unobserved prospective audit was completed; the results of which can be seen in figure 4 .

Act

Acting on results from the audits and opinion poll within this improvement cycle, a further departmental 'roll out' was conducted in order to try and restore adherence to the SOP. Attempting to make these handover procedures, the new norm would likely reduce the risk of reduced adherence to the SOP during SHO changeover.

\section{PDSA cycle 3}

Plan

The initial survey identified that the SHO cohort wanted formal teaching on the contents of a safe and efficient handover. In response to this, the plan for the third PDSA cycle was to introduce a handover segment to the ED SHO departmental induction.

\section{Do}

A short handover talk was delivered at SHO induction. This talk was delivered by one of the QIP leads who introduced the new doctors to the improvement initiative and the methods we used. The national handover guidance underpinning this QIP was referenced, and the SHOs were taught how to create the electronic handover shortcut and what the components of a safe and efficient handover should be.

\section{Study}

As with previous interventions, we conducted an unobserved prospective audit of handover practices against the agreed standard; the results can be seen in figure 4 . While adherence to the SOP were partially maintained, there was another decrease in quality of handover practices coinciding with the ED SHO changeover. 


\section{Act}

With another fall in compliance to the handover SOP, additional strategies are being considered to maintain the quality of handover despite the changing ED SHO cohorts. This QIP did achieve improvement in the quality of the patient handover from the baseline measurement; however, the issue remains as to how to alleviate the drop in adherence when changing cohort.

\section{LESSONS LEARNT}

This QIP highlighted how standardisation and education can support an effective, efficient and safe handover of patients at the end of the ED SHO shift. Importantly, it also has demonstrated the difficulty in maintaining change and adherence to new local practices with the constantly rotating SHO cohorts. Each PDSA cycle instigated during this QIP promoted a degree of improvement in adherence to national guidance, and overall, the quality of handovers has significantly improved from the baseline measurement to the final blinded audit (figure 4).

A lesson from this QIP was that to achieve improvement, all major stakeholders needed to support and drive the initiatives forward. Throughout the QIP, one of the SHO QIP leads was always present in the department, as were the ED consultants supervising the improvements. As such, we were able to continue to promote change. The ED SHOs were asked early regarding their opinions on the handover process and how it could be improved, and the improvement strategies were chosen with consideration of the SHO's responses.

Several national healthcare bodies call for standardisation of the handover process to maximise patient safety. We implemented this national guidance at local level through modification of our existing medical record programme. A limitation of this QIP was the delay in producing a pre-existing handover note built into the electronic records system. As such, we chose to teach each SHO how to create a handover shortcut, as discussed in the design of PDSA cycle 2. Initially, this resulted in some heterogeneity and demanded formal training from one of the QIP leads to achieve the standardisation that subsequently lead to improvements in the handover process.

When comparing this QIP to those looking at the medical handover, where it is commonplace for a formal meeting to occur at the end of the day/night team's shift, improving handover in the ED demands strategies to alleviate the issues of shift work. There needs to be the same comprehensiveness as would be seen in a formal end of shift meeting, but on multiple occasions, and between doctors often of differing experiences. The interventions instigated in this QIP did show improvements; however, sustained adherence was difficult to achieve. One significant difference between end of shift handovers in the ED rather than on a ward/medical take is that the handovers in the ED do not have protected rota time. Additionally, the ED SHO rota was structured in such way that evening SHO changeover was at variable times, making individual meetings between outgoing and incoming SHOs the only option. A shift in ethos to introduce a protected time period during the last $30 \mathrm{~min}$ of the rotating ED SHO's shift may promote consistent change.

The main barrier to continued improvement identified during this QIP, as previously highlighted, was a drop in adherence to the improvement initiatives with each new rotation of ED SHOs. We attempted to overcome this through the introduction of a handover talk at the $\mathrm{SHO}$ induction, which did improve adherence. Further unobserved prospective audits are required to identify if the induction talk is maintaining the handover standards we aimed for.

\section{CONCLUSION}

Through an unobserved prospective audit alongside a subjective questionnaire, we identified significant shortcomings in the ED SHO patient handover. With concern that the handover process could compromise patient safety, several improvement strategies using the PDSA cycle structure were implemented. Overall, our interventions improved $4 / 5$ handover components tested and maintained a $100 \%$ adherence to the fifth component.

An important finding in this QIP was the difficulty in maintaining adherence to new interventions with a constantly rotating SHO cohort. We identified this limitation early and audited the handover practices immediately after the SHO changeover to confirm the issue, as well as through having a QIP lead who was able to champion effective handover across multiple SHO rotations. Our subsequent attempts to overcome this issue have shown early success; however, ongoing analysis of handover practice is needed to confirm persistent improvements.

An additional analysis would be to attempt to quantify the impact improving the handover had on patient care. A more robust handover process will certainly improve patient safety; however, demonstrating an actual improvement in patient care would be a more challenging study. Twitter Anu Mitra @AcmeDR

Contributors MS: idea, improvement implementation, data collection, data analysis, data presentation and manuscript synthesis. JG: improvement implementation, data collection, data analysis, data presentation and manuscript review. PK: QIP fellow, supervision, data analysis, data presentation and manuscript review. HH: improvement implementation, data collection and manuscript review. AM: QIP consultant, supervision, improvement implementation and manuscript review.

Funding The authors have not declared a specific grant for this research from any funding agency in the public, commercial or not-for-profit sectors.

Competing interests None declared.

Patient and public involvement Patients and/or the public were not involved in the design, or conduct, or reporting, or dissemination plans of this research.

Patient consent for publication Not required.

Provenance and peer review Not commissioned; externally peer reviewed.

Data availability statement All data relevant to the study are included in the article or uploaded as supplementary information.

Open access This is an open access article distributed in accordance with the Creative Commons Attribution Non Commercial (CC BY-NC 4.0) license, which 
permits others to distribute, remix, adapt, build upon this work non-commercially, and license their derivative works on different terms, provided the original work is properly cited, appropriate credit is given, any changes made indicated, and the use is non-commercial. See: http://creativecommons.org/licenses/by-nc/4.0/.

\section{REFERENCES}

1 British Medical Association. NHS pressures, 2017.

2 Frey R, Decker K, Reinfried L, et al. Effect of rest on physicians' performance in an emergency department, objectified by electroencephalographic analyses and psychometric tests. Crit Care Med 2002;30:2322-9.

3 NHS England. Transforming urgent and emergency care services in England, 2015. Available: https://www.england.nhs.uk/wp-content/ uploads/2015/06/trans-uec.pdf [Accessed 29 Sep 2018].

4 Junior Doctors Committee. Safe handover: safe patients. Guidance on clinical handover for clinicians and managers, 2005.

5 Royal College of Physicians. Acute care toolkit 1: handover. London: Royal College of Physicians, 2011. https://www.rcplondon.ac.uk/ guidelines-policy/acute-care-toolkit-1-handover

6 Curtis O, Fisher R. Improving medical SHO weekend handover at a tertiary referral centre. BMJ Qual Improv Rep 2013;2:u697.w971-9.

7 Raduma-Tomàs MA, Flin R, Yule S, et al. Doctors' handovers in hospitals: a literature review. BMJ Qual Saf 2011;20:128-33.

8 Till A, Sall H, Wilkinson J. Safe Handover : Safe Patients - The Electronic Handover System. BMJ Qual Improv Rep 2014;2:u202926. w1359.
9 Farhan M, Brown R, Woloshynowych M, et al. The ABC of handover: a qualitative study to develop a new tool for handover in the emergency department. Emerg Med J 2012;29:941-6.

10 Dawdy MR, Munter DW, Gilmore RA. Correlation of patient entry rates and physician documentation errors in dictated and handwritten emergency treatment records. Am J Emerg Med 1997:15:115-7.

11 Heller RA, Hu L. Making the weekend work: a local quality improvement project to establish and improve the quality of weekend handover. BMJ Open Qual 2018;7:e000215.

12 Maroo S, Raj D. Introducing a new junior doctor electronic weekend handover on an orthopaedic ward. BMJ Qual Improv Rep 2017;6:u212695.w5059.

13 Govier M, Medcalf P. Living for the weekend: electronic documentation improves patient handover. Clin Med 2012;12:124-7.

14 Vithlani R. Introduction of a new electronic medical weekend handover at Tunbridge wells Hospital. BMJ Qual Improv Rep 2015;4:u204755.w2342.

15 Kostelec P, Emanuele Garbelli P, Emanuele Garbelli P. Introduction of a Microsoft Excel-based unified electronic weekend handover document in acute and general medicine in a DGH: aims, outcomes and challenges. BMJ Qual Improv Rep 2017;6:u212152. w5721.

16 Cerner hospitals and health systems. Available: https://www.cerner. com/solutions/health-systems

17 Safer Care. SBAR. Available: https://www.england.nhs.uk/ improvement-hub/wp-content/uploads/sites/44/2017/11/SBARImplementation-and-Training-Guide.pdf 